\title{
Wireless Signal Transmission and Excitation for Piezoelectric Active Fibre Composite Elements
}

\author{
Christian Dürager ${ }^{1}$, Andreas J. Brunner ${ }^{1}$ \\ 1. Empa, Swiss Federal Laboratories for Material Testing and Research, Dübendorf, Switzerland
}

\begin{abstract}
This paper presents an application for wireless structural health monitoring (SHM) in composite structures with piezoelectric Active Fibre Composite (AFC) elements. Previous studies have shown the potential of piezoelectric AFC elements used as sensor or emitter for structural waves in SHM applications. The objective of the present study was to demonstrate the functionality of a piezoelectric AFC element used as wireless sensor. The wireless sensor consists mainly of two parts. For measuring changes in the impedance of the composite structure, a piezoelectric AFC element is used. A simple electric circuit transmitted the measured signals wirelessly to a receiver outside the structure thus eliminating the need for wire connections and the related decrease in mechanical integrity. Also, a wireless connection to a sensor network is feasible. For the experiments, the piezoelectric AFC element is surface bonded on a composite plate, a laboratory scale model system, and connected to a wireless transmitter. The AFC on the composite plate is stimulated by sinusoidal voltage of $100 \mathrm{kHz}$. Damage in the plate is simulated by mass loading at different locations. Measurement of the charges created by the piezoelectric AFC element during the stimulation provides the technical basis for detection of changes in the composite plate.
\end{abstract}

Key words: wire-less sensor modules, structural health monitoring, electrical impedance testing, piezoelectric active fibre composite, feasibility study

\section{INTRODUCTION}

Any attempt at up-scaling sensor networks for structural health monitoring (SHM) based on planar piezo-electric elements from laboratory scale to engineering structures is facing a number of problems. First, the larger size of the structures typically leads to an increase in the number of sensor elements, and most of the time of the wires (typically two per element) that have to be mounted, as long as the structure shall still be fully monitored. Second, the electrical capacitance of the piezoelectric elements either limits the total length of the wires between sensor and data acquisition, or requires the use of amplifiers in between. Third, if the sensor network and its components have to be protected against environmental exposure, including accidental impact, length and number of wires between element and data acquisition, or a suitable connecting point on the structure, may lead to considerable modification of the structure, in particular its surface, with a corresponding change of properties, appearance, or behavior. While the concept of distributed sensor networks [1] does reduce the number of wires, it does not address the problem of limited electrical capacitance and signal amplification, and, on the other hand, deletes the information on sensor position, unless specific measures or analysis procedures are introduced to identify individual sensors. Wire-less piezoelectric sensor modules, i.e., electric resonance circuits containing a piezoelectric element, are hence a promising approach for "large" sensor networks for SHM. Both, implementation of long and numerous wires, as well as of amplifiers can be avoided, while identification of individual sensor modules is feasible using well established radio frequency identification (RFID) techniques. The only drawback is the energy supply necessary for wire-less data transmission, and eventually, for data storage on the module. The present paper does explore the feasibility of using wire-less piezoelectric sensor modules for SHM. As a first step, wire-less signal transmission

Corresponding author:

C. Dürager, christian.duerager@empa.ch 
is attempted over relatively short distances, e.g., a few centimeters, only. This would be typical, e.g., for surface mounted sensor modules with some protection against environmental exposure or impact on top.

\section{EXPERIMENTAL}

\subsection{Principle of measurement}

The SHM-sensor used in the experiments is a socalled Active Fiber Composite (AFC) made from commercially available piezoceramic fibers sandwiched between interdigitated electrodes that have been screen-printed on foils [2]. This sensor element manufactured at Empa based on a design developed by Bent et al. [3] has an active area of $30 \mathrm{~mm} \times 20 \mathrm{~mm}$, a thickness of about $0.3 \mathrm{~mm}$, a capacitance of about 0.8 to $1.0 \mathrm{nF}$ (at $1 \mathrm{kHz}$ ), and is conformable to curved surfaces. It is mounted on the surface of the structure to be monitored with a commercial two-component adhesive. Piezoelectric AFC elements have been investigated as sensors for various nondestructive test (NDT) methods [4, 5]. For starting, electrical impedance testing has been selected as NDT method for evaluating the wireless AFC-sensor module. The electrical impedance of the AFC in the "free" state, i.e. not mounted or coupled to a structure essentially behaves as an electrical capacitance, except for certain frequencies that correspond to resonances. These resonance frequencies can be calculated from the effective fiber length of the elements.

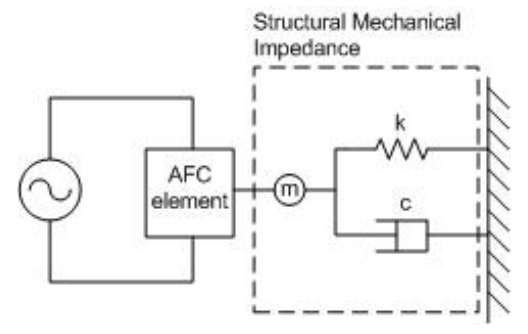

Figure 1: One dimensional model used to represent an AFC-driven dynamic structural system

When the AFC is coupled to a structure and supplied with an alternating voltage of a given frequency, the AFC vibrates and hence excites me- chanical waves in the structure. The measured electrical impedance of the coupled AFC-structure system then reflects the dynamic interaction between sensor and structure. Such a system can be described by an electro-mechanical model [6] schematically shown in Figure 1. This model is called a spring-mass-damper system where $m$ represents the mass, $c$ the damping coefficient and $k$ the spring constant. The coupling between electrical and mechanical impedance is calculated as follows:

$$
\begin{gathered}
\frac{1}{Z(\omega)}=Y(\omega) \\
Y(\omega)=i \omega \alpha\{\underbrace{\varepsilon_{33}^{-}(1-i \delta)}_{\text {Capacitance AFC }}- \\
-\underbrace{\frac{Z_{s}(\omega)}{Z_{s}(\omega)+Z_{\text {AFC }}(\omega)} d_{3 x}^{2} \widehat{Y}_{x x}^{2}}_{\text {Admittance Structure }}\}
\end{gathered}
$$

where $Z(\omega)$ represents the electrical impedance, $Y(\omega)$ the electrical admittance, $Z_{A F C}(\omega)$ the mechanical impedance of the AFC element, $Z_{s}(\omega)$ the mechanical impedance of the structure, $\varepsilon_{33}{ }^{\prime}$ the dielectric constant at zero stress, $\widehat{Y}_{x x}^{2}$ the complex Young's modulus, $d_{3 x}^{2}$ the piezoelectric coupling constant, $\omega$ the radian frequency, $\alpha$ the geometric constant of the AFC, $\delta$ the dielectric loss tangent of the AFC, and $i$ means $(-1)^{1 / 2}$.

\subsection{Device for wireless signal transmission}

The electronic device that provides the wire-less functionality for the piezoelectric AFC sensor consists mainly of two parts, one for signal processing and one for signal transmission as shown in Figure 2. The key point for using a piezoelectric AFC element to detect defect accumulation or growth in a structure is the change in the electro-mechanical impedance and the change of the current $I_{1}$ which flows into the AFC element. For detecting the value of the current $I_{1}$ the voltage drop $V_{m}$ on the resistor $R_{m}$, which is serial to the AFC element, is supplied to the differential amplifier. The output voltage of the differential amplifier $V_{0}$ is the sum of $V_{1}$ and $V_{2}$ and is obtained as follows:

$$
V_{0}=\left(V_{1}-V_{2}\right) \cdot \frac{R_{1}}{R_{2}}=V_{1}-V_{2}=V_{m}
$$




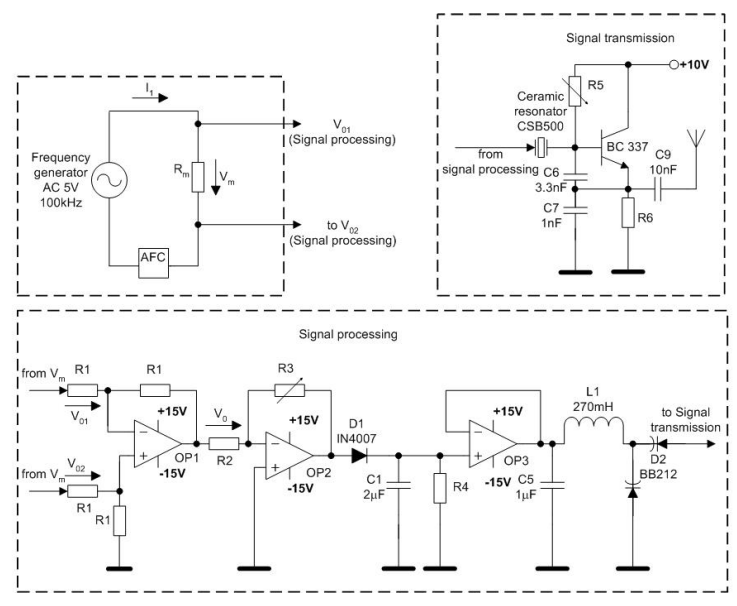

Figure 2: Equivalent circuit diagram of the wireless signal transmission device

where $V_{1}$ represents the voltage on the inverting input terminal, and $V_{2}$ the voltage to the noninverting terminal of the operational amplifier OP1. The inverting operational amplifier OP2 increases the voltage depending on the amplification factor which is calculated as follows:

$$
V_{01}=-V_{0} \cdot \frac{R_{3}}{R_{2}}
$$

where $V_{0}$ represents the voltage from the differential amplifier, $V_{01}$ the voltage after the inverting amplifier and the resistors $R_{2}$ and $R_{3}$ the voltage divider. Since a potentiometer was chosen for the resistor $R_{3}$ it is possible to change the gain of the inverting amplifier for the optimal voltage $V_{01}$. The diode D1 with the parallel capacitor rectifies the alternating current (AC) voltage to direct current (DC) voltage which is needed for the capacitor Diode D2. The impedance transformer OP3 in series with the inductor L1 decouples the signal processing part from the signal transmitting part of the electronic circuit. The value of the capacitance diode depends on the change of the voltage value which is supplied from the signal processing part after the amplifier OP3. For the transmitting circuit a Colpitts type oscillator with a ceramic resonator (type CSB 500) is used. Details of the Colpitts oscillator circuit are described in the literature (see, e.g. [7]). The oscillating frequency of this circuit decreases with increasing capacitance of the variable capacitance diode D2 and its capacitance decreases with the increasing output voltage $V_{01}$. Hence, when the current $I_{1}$ through the AFC element changes the voltage drop $V_{m}$ through $R_{m}$ this results in a change of the frequency of the oscillator. This allows detecting a variation of the electro-mechanical impedance by different values of the transmitted frequency.

\section{MEASUREMENT AND RESULTS}

\subsection{Measurement and setup}

A glass-fiber reinforced polymer (GFRP) epoxy plate $400 \mathrm{~mm} \times 400 \mathrm{~mm}$ with a thickness of $3 \mathrm{~mm}$ serves as model structure for the experiment. The GFRP plate was supported on three styrofoam pyramids indicated in Figure 4 and Figure 5. The choice of a composite material for wire-less experiments is arbitrary; the AFC element could be mounted on structures made of other materials. In the experiments reported in this paper, it was considered important to use a "reversible" defect for the impedance measurements, i.e., a change in mechanical impedance of the GFRP plate that could be applied, removed, and reapplied an arbitrary number of times without inducing a permanent change. It was hence decided to use a variable

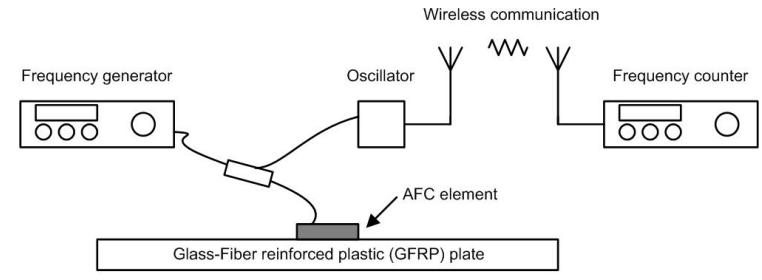

Figure 3: Schematic set-up for the measurement system with wire-less signal transmission.

weight, consisting of a wooden block (dimensions $185 \mathrm{~mm} \times 30 \mathrm{~mm} \times 60 \mathrm{~mm}$ ) with bore-holes for additional metal weights (screws). This block could be put on the GFRP plate in arbitrary locations (see Figure 5), and its mass varied (920g or 1690g) 
by varying the number of screws. It has to be noted that the weight of the block is nearly comparable to or exceeding that of the GFRP plate (935g), quite likely resulting in an artificially high change in mechanical impedance. Further experiments will be performed with smaller changes in mechanical impedance, in order to determine the limits of sensitivity of the AFC-sensor.

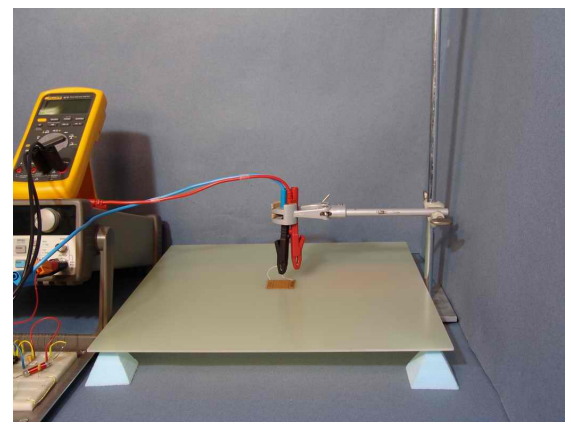

Figure 4: Photograph of the setup.

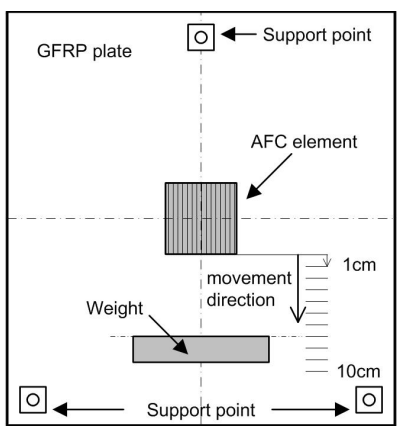

Figure 5: Schematic of the GFRP-plate with surface bonded AFC as model structure for SHM.

In order to analyze the change of the electro-mechanical impedance depending on the location of the damage, the weight is shifted away from the AFC element in steps of $1 \mathrm{~cm}$ (Figure 5). The electronic device (section 2.2.) sends this change wirelessly as frequency dependent signal to the frequency counter (Figure 3).

\subsection{Preliminary measurements}

In the first step, the voltage drop $V_{m}$ on the resistor $R_{m}$ as function of the distance of a weight (metal plate of $3000 \mathrm{~g}$ with an area of $185 \mathrm{~mm} \mathrm{x}$ $30 \mathrm{~mm}$ ) from the AFC (Figure 5) was measured. The alternating voltage was supplied by a com-

\begin{tabular}{|c|c|c|}
\hline Displ. $[\mathrm{cm}]$ & $V_{m}[\mathrm{mV}]$ & Freq. $[\mathrm{kHz}]$ \\
\hline \hline 1 & 574 & 512 \\
2 & 572 & 516 \\
3 & 571 & 518 \\
4 & 571 & 520 \\
5 & 569 & 523 \\
6 & 569 & 533 \\
7 & 567 & 541 \\
8 & 565 & 552 \\
9 & 566 & 560 \\
\hline
\end{tabular}

Table 1: Voltage drop $V_{m}$ and corresponding change in frequency as a function of the distance of the weight of $3000 \mathrm{~g}$ from the AFC element from the preliminary tests.

mercial frequency generator (type HP 33120A) and the voltage $V_{m}$ was measured with a multimeter (type KEITHLEY 195 System DMM). In a second step, the frequency dependence of the voltage drop $V_{m}$ was determined with an oscilloscope (type TDS 210 from Tektronix) as shown in figure 3. The results of both measurements are compiled in table 1 .

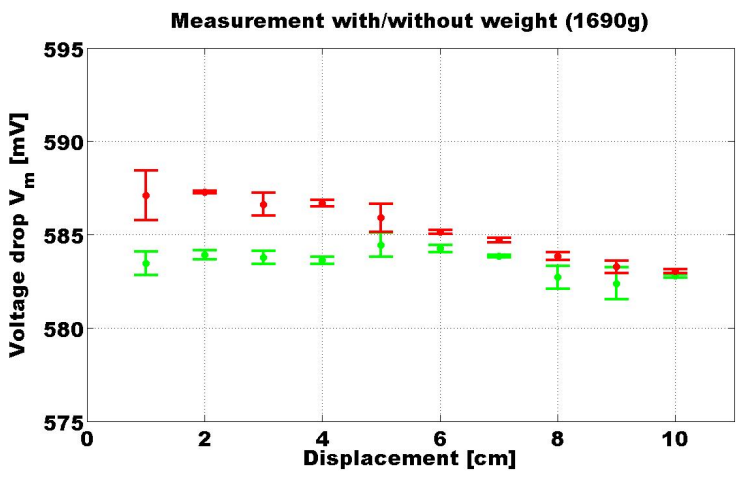

Figure 6: Measured voltage drop $V_{m}$ versus distance of the weight of 1'690 $\mathrm{g}$ from the AFC element on the GFRP plate. The red data points indicate averages of five measurements each; the bars represent the standard deviations, the green data points are the corresponding values after removal of the weight (see text for details). 


\subsection{Varying location of "reversible" defect}

A series of systematic measurements was then performed with the weights described in section 2.3, Figure 6 summarizes the results for the weight of $1690 \mathrm{~g}$ as a function of distance from the AFC element. Each data point represents an average of five measurements for each of the two conditions "weight applied" and "weight removed". The standard deviations vary considerably, but typically amount to roughly $+/-1 \mathrm{mV}$. The comparison of the curves in Figure 6 shows that the two conditions can clearly be distinguished (separated by more than one standard deviation) up to distances of about $8 \mathrm{~cm}$ from the AFC element. It has to be noted that equilibration of the GFRP plate after application and removal of the weight took time. For the data shown in Figure 6, the readings were taken consistently, i.e., 2 minutes after application and removal of the weight, respectively.

\subsection{Varying mass of "reversible" defect}

Repeating the measurements with a weight of 920 $\mathrm{g}$ (lower than the mass of the model structure) indicated that the separation between the two conditions "weight applied" and "weight removed" decreased for comparable distances from the AFC element (data not shown). A separation by more than one standard deviation (again five measurement per data point) was not achieved anymore.

\section{DISCUSSION}

From the literature (see, e.g. [8]) and from electrical impedance measurements as a function of frequency using AFC elements bounded on GFRP plates (unpublished results) it is known that the impedance spectra as a function of frequency show pronounced peaks at certain frequencies for a given damage state of the structure. For the wire-less measurements, the excitation was chosen as $5 V_{p p}$ at a frequency of $100 \mathrm{kHz}$. Hence, the changes in impedance observed upon applying the model damage (wooden block) to the GFRP plate do not necessarily correlate with peaks in the impedance spectrum related e.g. to specific plate modes. Changes in these peaks (either in intensity or as frequency shifts) do seem to indicate changes in the structure with higher sensitivity than changes in the overall (typically $1 / \mathrm{f}$ behavior) of the impedance spectra as a function of frequency. It is expected that tuning the excitation frequency to a value of a suitable peak, or by applying a range of excitation frequencies consecutively, i.e. a sweep, the separation of different plate conditions could be improved considerably.

\section{OUTLOOK}

As noted earlier, it is desirable to apply a reversible defect, however, one that yields "realistic" stiffness changes, due to e.g. crack formation or delamination growth. Application and removal of this "defect" should result in negligible mass changes for further experimentation on the GFRP plate. It would also be desirable to simulate a range of stiffness changes and of defect locations for a given change in stiffness. It is important to note that electrical impedance measurements have been chosen as one example for SHM with AFC. The AFC elements, however, can be used as sensors for other NDT methods as well (e.g., acoustic emission, AE) or as sensor-emitter pairs for acousto-ultrasonics (AU) or guided waves. With the existing electronic device for wire-less signal transmission it is straight-forward to investigate wire-less signal transmission from AFC elements used as AE sensors on the GFRP plate subject to simulated AE, e.g. lead pencil breaks, known as Hsu-Nielsen sources [9]. Another approach would be to develop wire-less excitation of AFC elements to be used as emitters for, e.g. AU.

\section{Acknowledgment}

The technical assistance of Mr. H. Huber (Electronics/Metrology/Reliability Laboratory of Empa) is gratefully acknowledged. The project has been supported by a grant from the 5th series of Research and Development projects at Empa.

\section{References}

[1] M.J. Sundaresan, M.J. Schulz, A. Ghoshal, J. Intell. Mat. Syst. Struct. Vol. 12, No. 10 (2001), pp. 689-699.

[2] A.J. Brunner, M. Barbezat, C. Huber, P. Flüeler, Mater. \& Struct., Vol. 38, No. 279 (2005), pp. 561-567.

[3] A.A. Bent and N.W Hagood, J. Intell. Mat. Syst. Struct. Vol. 8, No. 11 (1997), pp. 903-919. 
[4] A.J. Brunner, M. Birchmeier, M. Barbezat, Proc. of AAUM 2006, Symposium on Advanced Acoustic Emission and Ultrasonic and/of New Materials, Kanegawa, Japan, Ed. M. Takemoto (2006), pp. 25-32.

[5] M. Barbezat, A.J. Brunner, Ch. Huber, P. Flüeler, J. Intell. Mat. Syst. Struct. (2007), at press.

[6] C. Liang, F.P. Sun, C.A. Rogers, J. Intell. Mat. Syst. Struct, Vol. 5, (1994), pp. 12-20.

[7] Li Guo-Hui, Chaos, Solitons \& Fractals, Vol. 26, No. 1 (2005), pp. 87-93.

[8] D.M. Peairs, G. Park, D.J. Inman, J. Intell. Mat. Systs. and Struct. Vol. 15, No. 2 (2004), pp. 129-139.

[9] R.K. Miller, E.v.K. Hill, P.O. Moore, Nondestructive Testing Handbook, Vol. 6, Acoustic Emission Testing, American Society for Nondestructive Testing (2005), p. 133. 